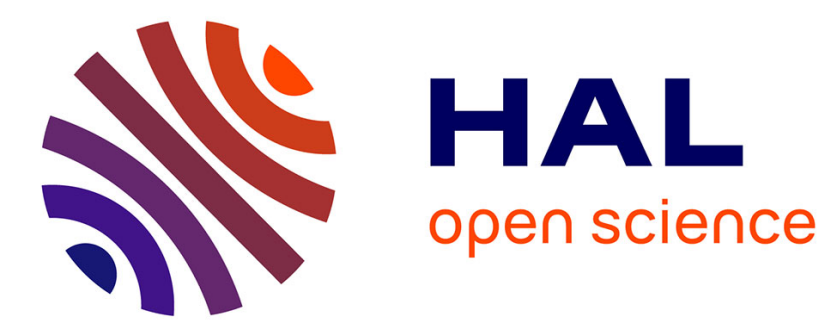

\title{
A propos de la statue portrait du roi Jayavarman VII au temple de Préah Khan de Kompong Svay
}

Christophe Pottier

\section{To cite this version:}

Christophe Pottier. A propos de la statue portrait du roi Jayavarman VII au temple de Préah Khan de Kompong Svay. Arts Asiatiques, 2000, 55, pp.171-172. halshs-02043468

\section{HAL Id: halshs-02043468 \\ https://shs.hal.science/halshs-02043468}

Submitted on 21 Feb 2019

HAL is a multi-disciplinary open access archive for the deposit and dissemination of scientific research documents, whether they are published or not. The documents may come from teaching and research institutions in France or abroad, or from public or private research centers.
L'archive ouverte pluridisciplinaire HAL, est destinée au dépôt et à la diffusion de documents scientifiques de niveau recherche, publiés ou non, émanant des établissements d'enseignement et de recherche français ou étrangers, des laboratoires publics ou privés. 


\section{Persée}

http://www.persee.fr

\section{A propos de la statue portrait du roi Jayavarman VII au temple de Préah Khan de Kompong Svay}

\section{Christophe Pottier}

Pottier Christophe. A propos de la statue portrait du roi Jayavarman VII au temple de Préah Khan de Kompong Svay. In: Arts asiatiques. Tome 55, 2000. pp. 171-172.

Voir l'article en ligne

\section{Avertissement}

L'éditeur du site «PERSEE »- le Ministère de la jeunesse, de l'éducation nationale et de la recherche, Direction de l'enseignement supérieur, Sous-direction des bibliothèques et de la documentation - détient la propriété intellectuelle et les droits d'exploitation. A ce titre il est titulaire des droits d'auteur et du droit sui generis du producteur de bases de données sur ce site conformément à la loi n`98-536 du $1 \mathrm{er} \mathrm{juillet} 1998$ relative aux bases de données.

Les oeuvres reproduites sur le site «PERSEE » sont protégées par les dispositions générales du Code de la propriété intellectuelle.

Droits et devoirs des utilisateurs

Pour un usage strictement privé, la simple reproduction du contenu de ce site est libre.

Pour un usage scientifique ou pédagogique, à des fins de recherches, d'enseignement ou de communication excluant toute exploitation commerciale, la reproduction et la communication au public du contenu de ce site sont autorisées, sous réserve que celles-ci servent d'illustration, ne soient pas substantielles et ne soient pas expressément limitées (plans ou photographies). La mention Le Ministère de la jeunesse, de l'éducation nationale et de la recherche, Direction de l'enseignement supérieur, Sous-direction des bibliothèques et de la documentation sur chaque reproduction tirée du site est obligatoire ainsi que le nom de la revue et- lorsqu'ils sont indiqués - le nom de l'auteur et la référence du document reproduit.

Toute autre reproduction ou communication au public, intégrale ou substantielle du contenu de ce site, par quelque procédé que ce soit, de l'éditeur original de l'oeuvre, de l'auteur et de ses ayants droit.

La reproduction et l'exploitation des photographies et des plans, y compris à des fins commerciales, doivent être autorisés par l'éditeur du site, Le Ministère de la jeunesse, de l'éducation nationale et de la recherche, Direction de l'enseignement supérieur, Sous-direction des bibliothèques et de la documentation (voir http://www.sup.adc.education.fr/bib/ ). La source et les crédits devront toujours être mentionnés. 


\section{A propos de la statue portrait du Roi Jayavarman VII au temple de Préah Khan de Kompong Svay}

Durant les derniers jours de janvier 2000, à l'occasion d'une expédition au Préah Khan de Kompong Svay avec le professeur Claude Jacques, conseiller auprès de l'UNESCO, et Mlle Ashley Thompson, conseillère à APSARA, j'ai eu la bonne fortune de repérer deux éléments de statues dont l'un pouvait correspondre au torse de la statue portrait du roi Jayavarman VII, dont la tête, particulièrement célèbre, provenant de ce temple est actuellement conservée au Musée National de Phnom Penh ${ }^{1}$. Ce torse était situé à une vingtaine de mètres à l'extérieur de l'angle sud-est de la première enceinte, en contrebas d'une terrasse périphérique en latérite (fig.1), mais il est probable qu'il provient d'une excavation peu éloignée, réalisée à l'occasion du pillage intense qui a eu lieu jusqu'en 1999 dans, et autour de la première enceinte. La tête, lors de sa découverte le 12 janvier 1958, était située dans la première enceinte « au pied et à l'est de la tour centrale $»^{2}$. Il n'est donc pas interdit de penser que le corps provienne de cette tour et que d'autres fragments puissent être trouvés dans le futur dans cette zone jonchée d'un amas confus de blocs.

Bien que ce torse se limite au tronc de la statue, puisque les épaules, le bas du fessier et la totalité des cuisses manquent, ses dimensions ${ }^{3}$, ses caractéristiques morpho- logiques, la qualité remarquable de la facture, le grès dense gris-vert et d'aspect légèrement marbré dans lequel il a été taillé et la cassure du cou, permettaient de faire rapidement le rapprochement entre ce tronc et la fameuse tête du Musée National. La réalisation d'un moulage de la cassure du cou ayant permis de confirmer cette identification, la Conservation d'Ang-
Statue portrait du roi Jayavarman VII, grès, fin $\mathrm{XII}^{\mathrm{e}}$-début $\mathrm{XIII}^{\mathrm{S}} \mathrm{s}$., Cambodge Fig. 1 (à gauche) Torse, H. $70 \mathrm{~cm}$, trouvé à Kompong Svay Fig. 2 (à droite) Tête (Musée national de Phnom Penh, $\mathrm{n}^{\circ}$ 989) temporairement assemblée au torse 
kor en collaboration avec l'EFEO a rapatrié le torse au Musée National le 30 mars. Lassemblage avec la tête a aussitôt été réalisé par l'atelier de restauration sous la direction de Bertrand Porte ${ }^{4}$ pour que l'ensemble soit temporairement exposé lors de la Journée du Patrimoine le 3 avril (fig. 2).

La découverte de ce torse permet de compléter - en partie seulement - la statue portrait de Jayavarman VII provenant du Préah Khan de Kompong Svay, et de redonner ainsi une échelle humaine à cette tête qui, à force d'être reproduite en moulage ou en photographie, tend à être perçue comme une icône quasi autonome. Plus pragmatiquement cette découverte répond, quarante ans après, à l'attente de G. Cœedès qui souhaitait dans ces colonnes que ce corps fût retrouvé pour éventuellement confirmer I'hypothèse de P. Stern selon laquelle «l'impression de jeunesse qui se dégage du visage" de Jayavarman VII est "presque la preuve» que ce dernier a "vécu durant sa jeunesse" au Préah Khan de Kompong Svay, pouvant corroborer du même coup l'attribution de la fondation de ce temple à Jayavarman VII ou à son père (Cœedès, 1960 : 191-193. Stern, 1965: 98, 150, 173-174) ${ }^{5}$. En fait, il me semble que les caractéristiques morphologiques du torse découvert ne different guère des autres exemples connus et, à moins d'envi- sager une prise d'embonpoint précoce du prince, on doit-je crois - abandonner cette idée d'une statue de Jayavarman VII "beaucoup plus jeune».

Lors de ce récent séjour au Préah Khan de Kompong Svay, un second corps a été repéré sur une terrasse de latérite à l'ouest du gopura oriental de la troisième enceinte $^{6}$ : il s'agit d'un personnage masculin, acéphale, assis en méditation, en dhyāna-mudrã. les jambes en virasâna. vêtu d'un simple sampot à la ceinture ornée. Le corps et son socle rectangulaire non mouluré sont bien conservés et seules manquent la tête et la majorité du dos, du cou et des bras. Cette statue est de morphologie légèrement différente et de facture de moindre qualité que le torse de la statue portrait repérée: le corps semble plus droit. l'abdomen moins développé, les ornementations incisées de la ceinture sont irrégulières. Mais sauf ces différences et la présence des mains posées en méditation dans le giron, l'échelle de la statue, son traitement naturaliste et le rendu de la musculature rappellent les statues portraits de Jayavarman VII. Ce corps en méditation au Préah Khan de Kompong Svay fait d'ailleurs écho à la présence d'une pièce du même type ${ }^{7}$ à l'emplacement où a été trouvé le corps de la plus complète des statues portraits de Jayavarman VII, celle dite de "Krol Roméas»" ${ }^{8}$. Un autre site pourrait réunir aussi ces deux «types» de statues: il s'agit du Ta Prohm d'Angkor, où l'on observe à l'extérieur du gopura III oriental un corps en méditation, acéphale et très érodé par un probable séjour prolongé dans la douve voisine ${ }^{9}$, alors qu'un torse pouvant correspondre à une statue portrait est encore actuellement dans la troisième enceinte $^{10}$. La présence de ces Buddha en méditation dans des sites où ont été découvertes des statues portraits évoque une hypothèse formulée par $\mathrm{G}$. Códès qui avait proposé d'identifier plusieurs têtes de Buddha aux images de Jayabuddhamahānātha (1960 : 195-196) et de considérer l'existence de deux séries de statues : les statues portraits de Jayavarman VII et les Jayabuddhamahãnâtha qui présentaient le souverain en tant qu'homme en position de vénération chez la première, et sous l'apparence du Buddha en méditation chez la seconde. Mais la confirmation d'une telle hypothèse nécessiterait une étude plus détaillée que ne le permet cette présente note, en particulier un examen des exemplaires de ces deux types de statues dont plusieurs ont été repérés depuis 1960.

CHRISTOPHE POTTIER Architecte, Membre de l'EFEO Siemreap

\section{Notes}

1 Musée National de Phnom Penh. $\mathrm{n}^{\circ}$ 989. Dimensions : $40.5 \times 27 \times 28.5 \mathrm{~cm}$. Illustration dans : Jes sup \& Zéphir, 1997 : 89-90.

2 Coedès, 1960: 191. Rapport de la Conservation d'Angkor, décembre 1957-février 1958.

3 Dimensions: $71 \times 50 \times 41 \mathrm{~cm}$, tour de labdomer au-dessus de la ceinture $: 136 \mathrm{~cm}$.

4 Un contact parfait s'opère sur les $2 / 3$ des plans de cassure, laissant une lacune importante à l'arrière et des éclats en périphérie. Le maintien de la tête a été assuré par un goujon en inox amovible logé dans une fourrure en résine epoxy.

5 B.P. Groslier revendique l'origine de la premièr partie de cette hypothèse $\left(1973: 152 \& n^{\circ} 1\right)$ mais s'oppose à l'attribution de la fondation de ce temple à Dharanindravarman II $(1973: 148$ \& 258). Plusieurs éléments suggèrent même l'existence d'une fondation primitive antérieure at xue siècle. Dans les années 60 , le corps avait fait l'objet de recherches mais celles-ci étaient restées d'une ampleur sans commune mesure avec les pillages récents qui y ont été perpétués (B.Dagens. communication personnelle).

6 Cette statue a été mise au jour dans le remblai, ou dans une fosse de cette terrasse probablement tardive, en décembre 1999. Elle a été rapatríé au Musée National où elle est désormais conservée sous le $n^{\circ} 2770$. Dimensions: 89 (sans tenon) x 87 x $62 \mathrm{~cm}$ : tour de l'abdomen au-dessus de la ceinture: $138 \mathrm{~cm}$.

7 Corps en méditation sur socle rectangulaire lisse. actuellement conservé au dépôt de la Conservation d'Angkor sous le n' DCA658 foú elle a été annotée récemment « Jayavarman VII $n$ ). Dimensions: 103.8 (sans tenon) $\times 89 \times 68 \mathrm{~cm}$, tour de l'abdomen au-dessus de la ceinture: $126 \mathrm{~cm}$. La tête, trouvée semble-t-il au même endroit et en même temps. n'a été identifíe que dans les années 60 . Elle avait alors été assemblée sur le corps: le raccordement paraissait correct, mais la partie arrière de la tête manquant, il ne concernait que la moitié du cou, d'où un léger doute qui avait conduit B.P. Groslier et B. Dagens à réaliser un montage «démontable». (B. Dagens, communication personnelle). La tête est conservée at Palais royal de Phnom Penh depuis 1994, et était précédemment au dépôt de la Conservation d'Angkor sous le n* DCA658A: H. 36: larg. $35 \mathrm{~cm}$.

8 Conservée depuis 1934 au Musée National de Phnom Penh, $\mathrm{n}^{*} 1703$ (anciennement DCA 659). Sur cette pièce célébre, $c f$. Porte. 1999. Illustration dans Jessup \& Zéphir, 1997: 89, fig. 1. Tour de l'abdomen au dessus de la ceinture: $133 \mathrm{~cm}$. Sur la localisation du corps: Rapport de la Conservation d'Angkor, octobre 1924; Journaux de fouilles de la Conservation d'Angkor, tome 5. pp. 50-52, plan de localisation p. 55. Sur celle de la tête: Rapport de la Conservation d'Angkor, juin 1931: Journaux de fouilles de la Conservation d'Angkor, tome 8. p. 197, $256 \& 259$.

9 Corps en méditation sur socle semi-circulaire. très érodé: $\mathrm{H}$. sans tenon: $83 \mathrm{~cm}$ environ: larg. $95 \mathrm{~cm}$, tour de labdomen au-dessus de la ceinture: $123 \mathrm{~cm}$.

10 Grès gris-vert légèrement marbré, H. 92; larg. $70 \mathrm{~cm}$, tour de l'abdomen au-dessus de la ceinture: $125 \mathrm{~cm}$.

\section{Bibliographie}

-G. Coedès. 1960: «Le portrait dans l'art khmer". Arts Asiatiques, vol. VIV/3, p. 179-198

-B.-P. Groslier, 1973 : Inscriptions du Bayon, Paris. EFEO (Mémoires archéologiques, 3 ).

- H.I. Jessup \& T. Zéphir (sous la dir. de), 1997: Ang. kor et dix siecles d'art khmer, Paris, Réunion des Musées Nationaux.

-B. Porte, 1999: «Restauration de la statue du Roi Jayavarman VII assis, compte rendu d'intervention», Musée National de Phnom Penh, document dactylographié.

- P. Stern, 1965: Les monuments khmers du style du Bayon et Jayavarman VII, Paris, Presses Universitaires de France Publications du Musée Guimet. Recherches et documents d'art et d'archéologie. IX). 